\title{
Open access endoscopy: is the lost outpatient clinic of value?
}

\author{
B.P. Saunders and P.N. Trewby
}

Department of Medicine, Darlington Memorial Hospital, Hollyhurst Road, Darlington, Co. Durham DL3 $6 H X, U K$

\begin{abstract}
Summary: To test the value of an outpatient visit in patients with dyspepsia, 79 patients considered suitable for open access endoscopy by their general practitioners were instead seen in the medical outpatient clinic first. In 35 patients immediate endoscopy was seen as an inappropriate investigation and 23 of these were spared endoscopy. In 11 patients important extra diagnoses were made in the clinic which would have been delayed or missed had the patients been sent straight for open access endoscopy. Sixty-eight per cent of patients, when asked by questionnaire, said they preferred to be seen in the clinic first rather than come for open access endoscopy. These results lend support to the traditional medical clinic appointment followed by endoscopy if and when appropriate rather than the open access endoscopy system.
\end{abstract}

\section{Introduction}

In recent years there has been a rapid expansion of open access upper gastrointestinal endoscopy services. ${ }^{1}$ The patient by-passes the traditional outpatient consultation and is sent by the general practitioner straight to the hospital for endoscopy, their first contact with hospital staff often being in the endoscopy suite. Analyses of such services, concentrating on waiting times and percentage of patients shown to have pathology have in general been favourable $e^{2,3}$ and there has been pressure for the development of this service from general practitioners and hospital gastroenterologists. ${ }^{4,5}$ However, these studies have disregarded the value or otherwise of the lost outpatient appointment. The aim of this study was to assess the value of this initial outpatient appointment in a district general hospital not at present offering open access endoscopy (OAE), looking at endoscopies saved, additional diagnoses made in the clinic and patients' preference.

\section{Patients and methods}

Local general practitioners (GPs) were informed of the study. They were asked when referring patients with dyspepsia to indicate in a separate letter whether they wished the patient to be seen in the clinic first or whether they would prefer OAE without a clinic appointment. In 79 out of 119 cases referred with dyspepsia, the GP preferred OAE. These 79 patients form the basis of this study.

Correspondence: P.N. Trewby, M.D., F.R.C.P.

Accepted: 15 April 1993
All were seen in the outpatient clinic within 3 weeks of referral and were seen without knowledge of the GP's preference for OAE. A full history was taken and the patients were examined by consultant (40 patients), or registrar (30 patients) or senior house officer (nine patients) under supervision of the consultant. Endoscopy and other investigations were carried out as clinically indicated. Those patients endoscoped were given a confidential questionnaire asking if they would rather have come straight for OAE or if they preferred being seen in the clinic first. Patients were followed for 12 months to check for any new findings.

At the end of this time an objective assessment was made by the authors as to whether the patient had gained tangible benefit from coming to the outpatient clinic taking into account endoscopies saved, extra diagnoses made and alterations to existing therapy in the clinic.

\section{Results}

Of the 79 patients included in the study, endoscopy was considered the appropriate initial investigation in only 39 . These patients were endoscoped within one week of their clinic appointment (see Figure 1). Of the 40 in whom endoscopy was not considered as the appropriate initial investigation, 13 were already asymptomatic on treatment when first seen. Arrangements were made for these patients to complete their treatment and to telephone the unit if and when they next developed symptoms to arrange endoscopy before starting treatment again. 


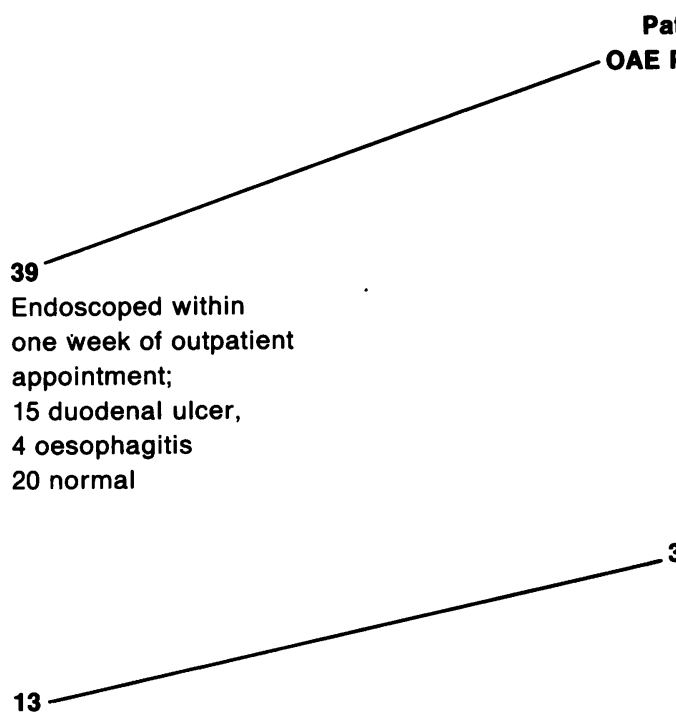

Asymptomatic when seen

in clinic on ulcer

healing treatment.

Endoscopy arranged

when next symptomatic

1

7

Endoscoped when symptomatic

(3 duodenal ulcer, 2 oesophagitis, 2 normal)

\section{6}

No further symptoms.

Not endoscoped

35 Not endoscoped immediately

Did not attend $\times 2$

Endoscopy not indicated as initial

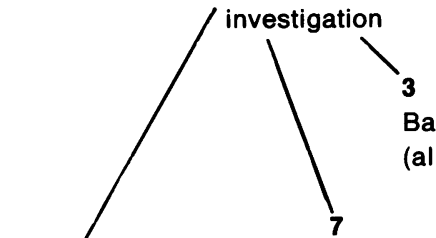

Barium studieso (all normal)

Symptoms not related to upper Gl tract

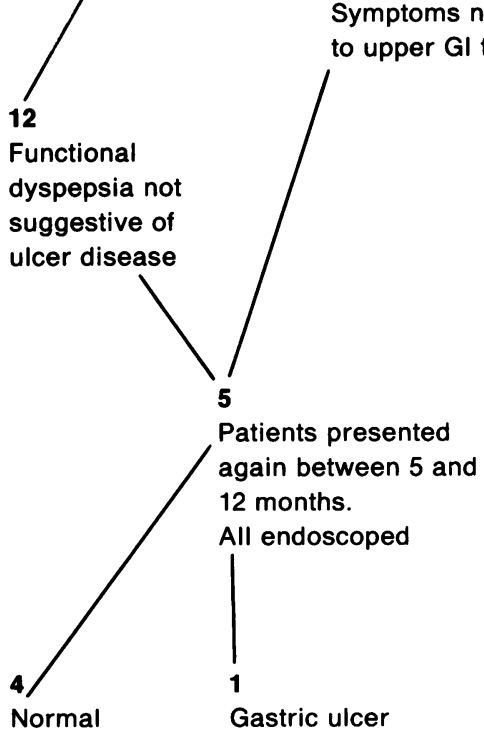

Figure 1 Outcome for patients in whom open access endoscopy was preferred by the patient's general practitioners. $\mathbf{G I}=$ gastrointestinal. 
Of these, seven have subsequently returned for endoscopy; three had duodenal ulcers, two had oesophagitis and in two no abnormality was found. Five of the remaining 40 patients not endoscoped did not attend the clinic on two or more occasions. In the remaining 22, endoscopy was not thought to be clinically indicated; in 12 of these, symptoms were thought to be functional in nature, in seven, symptoms were not directly related to the upper gastrointestinal tract, and in three, barium studies were considered a more appropriate initial investigation. Five of these 22 patients have since returned between 4 months and 1 year after their initial clinic appointment and all have since been endoscoped, and no abnormality found on endoscopy in four and a benign gastric ulcer in one who had presented initially with symptoms suggestive of irritable bowel syndrome.

In 11 patients important diagnoses were made in the outpatient clinic and treatment initiated which might have been delayed had the patient presented for OAE. One patient had colonic carcinoma, one primary biliary cirrhosis, one angina and one recurrent gastroenteritis. Seven had clinical depression as defined by remission of symptoms with antidepressant treatment and the development of no other pathology in the 12 month follow-up. None of these 11 patients was endoscoped. In three further patients the outpatient visit resulted in assessment and change of treatment of associated medical conditions (angina in two, chronic obstructive airways disease in one). In one case the wrong patient had been inadvertently sent to the clinic and would have been sent for OAE by his GP. The outpatient appointment allowed this mistake to be rectified.

When divided into those patients over and under 40 years of age, $33 \%$ of those under 44 (seven out of 21 patients) were thought to have benefited from being seen in the clinic first, and one diagnosis would have been delayed or missed if the patient had been sent for open access endoscopy, compared to $45 \%$ (26 out of 58) of those over 40 in whom 10 diagnoses might have been delayed or missed.

Of the patients endoscoped, $68 \%$ said they preferred to come to the clinic first. These patients were less likely to need time off work compared to those preferring OAE (29\% versus $66 \%)$ and less likely to have difficulty getting to the clinic (3\% versus $28 \%$, respectively). Despite a clinic waiting time of up to 2 hours only four patients felt the clinic waiting time was unacceptable.

\section{Discussion}

This study has shown the value of an outpatient appointment before endoscopy. Excluding five patients who did not attend, $29 \%$ of patients who would have been referred to OAE were spared an endoscopy altogether and the reduction in the number of endoscopies carried out would compensate financially for the extra clinic appointment and help reduce endoscopy waiting times. The use of a structured dyspepsia questionnaire might further reduce the number of patients needing endoscopy. ${ }^{6}$

In 13 patients the initial outpatient appointment allowed endoscopy to be timed logically for when the patient was next symptomatic off treatment. This resulted in three duodenal ulcers being picked up which may well have been missed had the patient presented for OAE at the time of their clinic appointment when they were asymptomatic. Important extra diagnoses were established in a further 11 patients during the outpatient visit and treatment of existing pathology altered in three. Against these advantages must be balanced a delay in diagnosis in one patient with benign gastric ulcer.

Overall $42 \%$ of our patients were thought to have gained tangible medical benefit from being seen in the clinic first and this was over and above advice about diet, smoking and alcohol given to all patients, and to the preparation of the patient for the endoscopy procedure itself and its likely result. Non-ulcer dyspepsia is the commonest single diagnosis in dyspeptic patients ${ }^{2}$ ( $36 \%$ in our group) and we feel counselling these patients as to the likely result of the endoscopy may considerably enhance the therapeutic role of the endoscopy in reassuring patients and helping them understand the mechanism of their systems.

Open access endoscopy is often advocated for patients aged over 40 with dyspepsia because of the risk of carcinoma. ${ }^{7}$ We certainly would not argue against endoscoping such patients with as short a waiting time as possible, but our results would favour a clinic treatment first because of the incidence of associated medical conditions, and the chance of assessing and counselling the patient. We were surprised but reassured that the majority of patients preferred a clinic appointment prior to endoscopy despite the need for two hospital visits and often prolonged waiting times. If patients in whom endoscopy was not carried out are included $81 \%$ are likely to have preferred the outpatient clinic first.

This study supports the classical outpatient appointment for patients with dyspepsia, with endoscopy being carried out if and when appropriate, rather than the open access endoscopy system.

\section{Acknowledgements}

We are indebted to Miss Julie Elliott for secretarial assistance. 


\section{References}

1. Bramble, M.G., Open-access endoscopy-a nationwide survey of current practice. Gut 1992, 33: 282-285.

2. Gear, M.W.L. \& Wilkinson, S.P. Open-acccess upper alimentary endoscopy. Br J Hosp Med 1989, 41: 438-444.

3. Hallisey, M.T., Fielding, J.W.L., Allum, W.H. \& Ellis, D.J. Managing dyspepsia. Lancet 1987 , i: $1533-1534$.

4. Macintyre, I.M.C. Open-access endoscopy for general practitioners. Practioner 1988, 232, 348-351.

5. Kerrigan, D.D., Brown, S.R. \& Hutchinson, G.H. Openaccess endoscopy: too much to swallow. Br Med J 1990, 300: 376-378.

6. Davenport, P.M., Morgan, A.G., Darnborough, A. \& DeDombal, F.T. Can preliminary screening of dyspeptic patients allow more effective use of investigational techniques. Br Med J 1985, 290: 217-220.

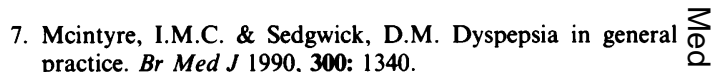

8. Fielding, J.W.L. The consequences of overlooking serious $\subseteq$ diseases of the upper gastrointestinal tract. In: Colin-Jones, D. $\overrightarrow{\vec{F}}$ \& Jones, R. (eds) Proceedings of a Symposium on Practical $\stackrel{\vec{T}}{+}$ Guidelines on the Management of Dyspepsia. Adelphi Com- 7 munication Limited, Macclesfield, 1990, pp. 28-33.

9. Health and Public Policy Committee, American College of $\frac{\overline{\bar{C}}}{\bar{D}}$ Physicians. Endoscopy in the evaluation of dyspepsia. Ann Intern Med 1985, 102: 266-269. 